\title{
AgroWeeder: Self-Driving Power-Weeder based on Raspberry Pi
}

\author{
Yash Sudhir Shirke \\ JSPM J.S. Polytechnic Hadapsar \\ Pune ,India
}

\author{
Prof. Mrs. Deepali S. Jadhav \\ JSPM J.S. Polytechnic Hadapsar \\ Pune ,India
}

\author{
Udayraj Sambhaji Gawade \\ JSPM J.S. Polytechnic Hadapsar \\ Pune, India
}

\begin{abstract}
The paper aim is to represent a Self-driving power AgroWeeder using the Raspberry-Pi. The pi-camera module along with an ultrasonic sensor is used to provide necessary data from the real world to the AgroWeeder which would then pass the data on to the raspberry-pi. The Agroweeder is capable of removing the weeds from the farm. This will help farmers to reduce their work of tilling the land and removing the weeds.
\end{abstract}

Keywords-AgroWeeder, Tiller, Raspberry pi

\section{INTRODUCTION}

With the growing needs of convenience, technology now tries to seek automation in every aspect possible. We present a self-driving Agroweeder which would eradicate human intervention in the field of driving. The Agroweeder would drive itself from one place to the other on its own it would possess integrated features like lane-detection, automatic weed remover, tilling of land, etc. This features would help the Agroweeder drive itself to the mentioned destination on the track properly, avid collisions, provide live streaming of the view in front of it with the help of camera mounted over the Agroweeder.

\section{HARDWARE REQURMENT}

\section{A. Raspberry pi $3 b+$ :}

- The Raspberry Pi three Model B+ is that the most well-liked Raspberry Pi laptop created, and therefore the Pi Foundation. Quicker processor, $5 \mathrm{GHz} \mathrm{WiFi}$, and updated LAN chip with Poe capability.

- The Raspberry Pi 3 Model B+ is that the newest product inside the Raspberry Pi 3 vary, boast Associate in Nursing updated 64-bit quad core processor running at one. $4 \mathrm{GHz}$ with intrinsically metal heatsink, dual-band two. $4 \mathrm{GHz}$ and $5 \mathrm{GHz}$ wireless computer network, quicker (300 mbps) LAN, and Poe capability via a separate Poe HAT.

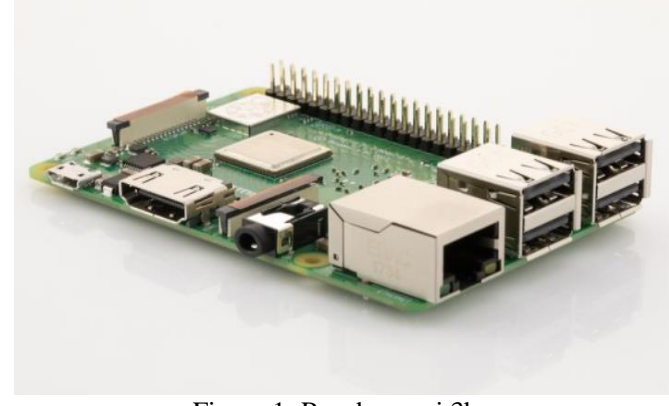

Figure 1: Raspberry pi 3b+

\section{B. Arduino uno R3:}

- Arduino can be a single-board microcontroller meant to form the applying tons of accessible that unit of measurement interactive objects and its surroundings. The hardware choices with Associate in Nursing ASCII document hardware board designed around Associate in Nursing 8-bit Atmel AVR microcontroller or a 32-bit Atmel ARM. Current models consists a USB interface, half-dozen analog input pins and fourteen digital I/O pins that permits the user to connect varied extension boards.The Arduino Uno board could be a microcontroller supported the ATmega328. it's fourteen digital input/output pins inside that six is employed as PWM outputs, a sixteen $\mathrm{MHz}$ ceramic resonator, Associate in Nursing ICSP header, a USB affiliation, half-dozen analog inputs, an influence jack and a push button.

- This contains all the \{required the desired\} support required for microcontroller. so as to induce started, they're merely connected to a laptop with a USB cable or with a AC-to-DC adapter or battery. Arduino Uno Board varies from all different boards which they will not use the FTDI USB-to-serial driver kick in them. it's featured by the Atmega16U2 (Atmega8U2 up to version R2) programmed as a USB-to-serial device.

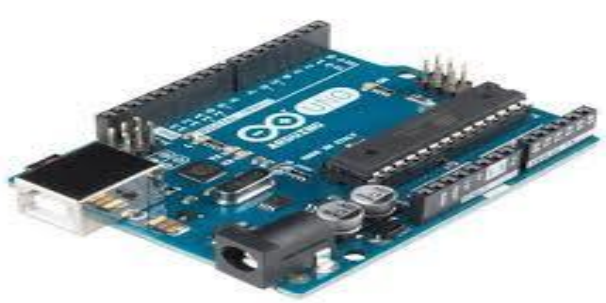

Figure 2: Arduino uno R3

C. Motor Driver (H Bridge):-

- The L298N can be a twin H-Bridge motor driver that allows speed and direction management of two DC motors at the same time. The module can drive DC motors that have voltages between 5 and $35 \mathrm{~V}$, with a peak current up to $2 \mathrm{~A}[2]$. The module has two screw terminal blocks for the motor A and $\mathrm{B}$, and another screw terminal block for very cheap pin, the VCC for motor and a $5 \mathrm{~V}$ pin which could either be Associate 
in Nursing input or output. this relies on the voltage used at the motors VCC[1].

- The module have Associate in Nursing on board 5V regulator that's either enabled or disabled using a jumper. If the motor supply voltage is up to $12 \mathrm{~V}$ we have a tendency to are able to alter the $5 \mathrm{~V}$ regulator and so the $5 \mathrm{~V}$ pin is employed as output, for instance for powering our Arduino board. however if the motor voltage is larger than $12 \mathrm{~V}$ we've got an inclination to ought to disconnect the jumper as a results of those voltages will cause damage to the on board $5 \mathrm{~V}$ regulator. during this case the $5 \mathrm{~V}$ pin are getting to be used as input as we'd like connect it to a $5 \mathrm{~V}$ power supply thus because the IC to work properly.

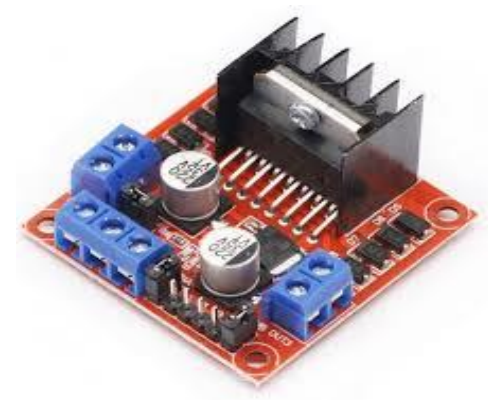

Figure 3: Motor Driver

D. Pi camera:-

- The Raspberry Pi Camera v2 is that the new official camera board free by the Raspberry Pi Foundation.

- $\quad$ The Raspberry Pi Camera Module v2 can be a chief quality eight megapixel Sony IMX219 image sensing element custom-made add-on board for Raspberry $\mathrm{Pi}$, that includes a hard and fast focus lens.

- The Raspberry Pi Zero presently comes complete with a camera port! victimization the new Raspberry Pi Zero Camera Adapter, you'll currently use a Raspberry Pi camera to your Zero

- It's capable of $3280 \times 2464$ constituent static photos, and additionally supports $1080 \mathrm{p}$ thirty, 720p sixty and 640x480p ninety video.

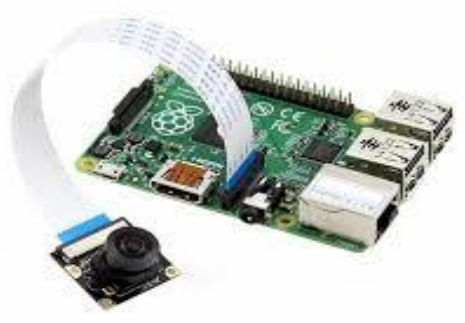

Figure 4: Raspberry pi camera
E. Ultra sonic sensor:-

- An quiet detector can be a tool which is able to live the house to Associate in Nursing object by exploitation sound waves. It measures distance by deed out a sound wave at a particular frequency and listening for that sound wave to recover.

- By recording the amount of your time between the sound wave being generated and so the sound wave bouncing back, it's possible to calculate the house between the measuring system detector and therefore the object.

\section{SOFTWARE REQUIMENTS}

A. python 3 IDE:-

- Python is one altogether those rare languages which could claim to be every straightforward and powerful. you will find yourself pleasantly aghast to see but straightforward it's to specialise in the solution to the matter rather than the syntax and structure of the language you are programming in.

- The official introduction to Python is Python could be a straightforward to be told, powerful artificial language.

- Its economical high-level information structures and a simple but effective approach to object-oriented programming. Python's elegant syntax and dynamic writing, in conjunction with its understood nature, build it an ideal language for scripting and quick application development in many areas on most platforms. ${ }^{[3]}$

\section{B. Arduino IDE:-}

- The Arduino integrated development setting (IDE) may be a cross-platform application (for Windows, macOS, Linux) that is written at intervals the artificial language $\mathrm{Java}^{[4]}$. it's accustomed write and transfer programs to Arduino compatible boards, but also, with the help of third party cores, completely different trafficker development boards.

- The American Standard Code for Information Interchange document for the IDE is discharged at a lower place the gnu General Public License, version 2. The Arduino IDE supports the languages $C$ and $\mathrm{C}++$ victimization special rules of code structuring. The Arduino IDE provides a computer code package library from the Wiring project, that has many common input and output procedures. User-written code alone desires two basic functions, for starting the sketch and conjointly the most program loop, that unit compiled Associate in Nursingd connected with a program stub main() into AN potential cyclic programme with the gnu tool chain, to boot boxed in with the IDE distribution.

- The Arduino IDE employs the program avrdude to convert the potential code into a file in number representation system secret writing that is loaded into the Arduino board by a loader program at intervals the board's coding system. 


\section{WORKING}

- The Agroweeder is a farm based machine which is used for tilling the land and the removing weeds from the land.

- The Agroweeder works on a simple small board processor raspberry pi $3 b+$. When the Agroweeder gets start the pi camera captures the live streaming of the field and sends the information to the raspberry pi.

- Then raspberry pi process the video, and sends the command to the arduino using GPIO pins.

- Then the arduino process the command and send the command to both the motor drivers.

- The first motor is use for running of Agroweeder.

- And the second one is used for controlling the weeding tools which is fixed behind the Agroweeder.

\section{CONCLUSTION}

Therefore our proposed idea will help farmers in saving their time and can earn more amount of money. They can operate our Agroweeder from home as well.

\section{REFERENCES}

[1] https://howtomechatronics.com/tutorials/arduino/arduino-dcmotor-control-tutorial-1298n-pwm-h-bridge/

[2] https://mytectutor.com/dc-motor-control-using-1298n-motordriver/

[3] https://www.cmi.ac.in/ madhavan/courses/prog22015/docs/python-3.4.2-docs-html/tutorial/index.html

[4] https://en.wikipedia.org/wiki/Arduino_IDE

[5] IEEE, 2014. www.ieee.org. [Online] Available at: http://www.ieee.org/about/news/2014/14_july_2014.html [Zugriff am 29 April 2015].

[6] Broggi, A. et al., 2013. Extensive Tests of Autonomous Driving Technologies. IEEE TRANSACTIONS ON INTELLIGENT TRANSPORTATION SYSTEMS, 14(3).

[7] J.M.A. Alvarez, A.M. Lopez \& R. Baldrich, IlluminantInvariant Model-Based Road Segmentation. Intelligent Transportation Systems, IEEE Transactions on, 12, 2008, pp 184-193.Bar Hillel, R. Lerner, D. Levi, \& G. Raz. Recent progress in road and lane detection: a survey. Machine Vision and Applications, Feb. 2012, pp. 727-745

[8] Shahzeb Ali Department of Electronic Engineering, Mehran University of Engineering \& Technology, Jamshoro, 2016 IEEE.

[9] K. R. Memon, S. Memon, B. Memon, A. R. Memon, and M. Z. A. S. Syed, "Real time Implementation of Path planning Algorithm with Obstacle Avoidance for Autonomous Vehicle," in 3rd 2016 International Conference on Computing for Sustainable Global Development”, New Delhi, India, 2016. 